\title{
Disoriented chiral condensate in presence of dissipation and noise
}

\author{
A. K. Chaudhuri: \\ Variable Energy Cyclotron Centre \\ 1-AF, Bidhan Nagar, Calcutta- 700064
}

\begin{abstract}
We have investigated the phase transition and disoriented chiral condensate domain formation in linear sigma model. Solving the Langevin equation for the linear $\sigma$ model, we have shown that for zero mass pions the fields undergo phase transition above a certain temperature $\left(T_{c}\right)$. For finite mass pions, there is no phase transition. It was also shown that when the fields, thermalised at temperature above $T_{c}$ are cooled down sufficiently rapidly, disoriented chiral condensate domains are formed quite late in the evolution, if the pions are assumed to be mass less. For massive pions, no large DCC domain is formed.
\end{abstract}

25.75.+r, 12.38.Mh, 11.30.Rd

\section{INTRODUCTION}

Equilibrium high temperature QCD manifest chiral symmetry if the quarks are assumed to be massless. At a critical temperature $T_{c}$, chiral symmetry is spontaneously broken by the formation of a scalar $\langle\bar{q} q\rangle$ condensate. In recent years there has been considerable excitement about the possibility of the formation of disoriented chiral condensate (DCC) domains [1 [7]. The basic idea is simple. In hadron-hadron or in heavy ion collisions, a macroscopic region of space-time may be created within which the chiral order parameter is not oriented in the same direction in the internal $O(4)=S U(2) \times S U(2)$ space as the ordinary vacuum. In heavy ion collisions some region can thermalise at temperature greater than $T_{c}$, the critical temperature for the chiral symmetry restoration. In that region the quark condensate $<\bar{q} q>$ vanishes. Once the system cools below $T_{c}$, chiral symmetry is spontaneously broken and the system evolve back to the true ground state where the condensate $\langle\bar{q} q>\neq 0$. Rajagopal and Wilczek [1 3 argued that in the non-linear environment (as will be the case in heavy ion collisions) as the temperature drops below $T_{c}$, the chiral symmetry may begin to break by developing domains in which the chiral field is misaligned from its true vacuum

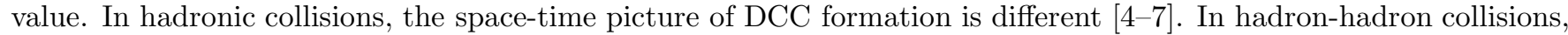
most of the models of particle production, stringy or partonic, put the bulk of the space-time activity near the light cone. The flow of produced quanta are concentrated in a rather thin shell expanding from the collision point at the speed of light. As the interior is separated from the true vacuum by rapidly expanding hot shell of partons, well inside it, quark condensate may be chirally rotated from its usual (vacuum) direction. This is the so called Baked-Alaska model of DCC production. The misaligned condensate has the same quark content and quantum numbers as do pions and essentially constitute a classical pion field. The system will finally relaxes to the true vacuum and in the process can emit coherent pions. Possibility of producing classical pion fields in heavy ion collisions had been discussed earlier by Anslem [8]. DCC formation in hadronic or in heavy ion collisions can lead to the spectacular events that some portion of the detector will be dominated by charged pions or by neutral pions only. In contrast, in a general event all the three pions $\left(\pi^{+}, \pi^{-}\right.$and $\left.\pi^{0}\right)$ will be equally well produced. This may then be the natural explanation of the so called Centauro events [9].

DCC phenomena may be easily detected in heavy ion collisions, if the collisions results in a single large DCC domain. Then comparing the measured spectra of neutral and charged pions on an event-by event basis, one could have identified the events dominated by DCC formation. The probability of a domain to yield a particular fraction (f) of neutral pions is $P(f)=1 / 2 \sqrt{f}$, provided all the isospin orientations are equally likely [4, 8, 10, 11. In contrast, a typical hadronic reaction without DCC, will produce a binomial distribution of $f$ peaked at the isospin symmetric value of $1 / 3$. Sizes of domains are then of great importance. A collision resulting in a single large domain can be easily identified from the ratio $\mathrm{f}$. On the otherhand, if the collision results in a number of small randomly oriented domains, the result will again be a binomial distribution, typical of a hadronic collision.

After the conjecture about DCC formation by Rajagopal and Wilczek [1 3 ] and also by Bjorken [4.5] several authors studied the phenomena [12 18. Microscopic physics governing DCC phenomena is not well known.It is in the regime of non-perturbative QCD as well as nonlinear phenomena, theoretical understanding of both of which are limited. One thus uses some effective field theory like linear $\sigma$ model with various approximations to simulate the chiral phase transition. Linear $\sigma$ model usually with Hartree type of approximation has been used extensively to simulate DCC phenomena [12,14,13]. In the linear sigma model chiral degrees of freedom are described by the the real $\mathrm{O}(4)$ field $\Phi=(\sigma, \vec{\pi})$. Because of the isomorphism between the groups $O(4)$ and $S U(2) \times S U(2)$, the later 
being the appropriate group for two flavor QCD, linear sigma model can effectively model the low energy dynamic of QCD [19]. Rajagopal and Wilczek [1] 3] used of quench initial condition to simulate the non-equilibrium evolution of the chirally symmetric field. Quench scenario assume that the effective potential governing the evolution of long wave length modes immediately after the phase transition at $T_{c}$ turns to classical one at zero temperature. It can happen only in case of very rapid cooling and expansion of the fireball. In heavy ion collisions quench like initial conditions are unlikely. Gavin and Mueller [13 considered the annealing scenario, in which the condensate evolve from $\langle\phi\rangle \sim 0$ to $\langle\phi\rangle \sim f_{\pi}$ slowly (as in the standard annealing) but in which the non-equilibrium conditions which allow amplification of long wavelength modes are maintained nevertheless. Explicit simulation with linear sigma model, indicate that DCC depends critically on the initial field configurations. With quench like initial condition DCC domains of 4-5 fm in size can form [14]. Initial conditions other than quench lead to much smaller domain size.

Very recently, effect of external media on possible DCC is being investigated [20 28]. Indeed, in heavy ion collisions, even if some region is created where chiral symmetry is restored, that region will be continually interacting with surrounding medium (mostly pions). Biro and Greiner [20] using a Langevin equation for the linear $\sigma$ model, investigated the interplay of friction and white noise on the evolution and stability of collective pion fields. In general friction and noise reduces the amplification of zero modes (they considered the zero modes only). But in some trajectories, large amplification may occur. We have also investigated the interplay of friction and noise on possible DCC phenomena 23 27. Simulation studies in $2+1$ dimension indicate that with quench like initial field configuration, large disoriented chiral condensate domains can still be formed. We have also performed a simulation in $3+1$ dimension. It was seen that with zero mass pions, even with thermalised fields as initial condition, DCC domains may be obtained, late in the evolution. However, as will be shown in the present paper, with finite mass pions, the different results are obtained. We find no DCC domain structure of appreciable size, even late at evolution.

In the present paper, we have investigated in detail the properties of phase transition in linear sigma model and subsequent DCC domain formation in $3+1$ dimension. The paper is organised as follows: in section 2 , we briefly describe the Langevin equation for linear $\sigma$ model and show that for zero mass pions, the model undergoes 2 nd order phase transition at finite temperature. For massive pions there is no exact phase transition in the model, though the symmetry is restored to a large extent at high temperature. In section 3 , growth of disoriented chiral condensate domains will be studied. It will be shown that while with zero pion mass, large DCC domains can form, with fast cooling law, for massive pions no such domains are formed. In section 4 , we will vindicate the results obtained in section 3, from study of pion-pion correlation. The probability distribution function for the neutral to total pion ratio will be obtained in section 4. Lastly, in section 5, summary and conclusions will be drawn.

\section{LINEAR SIGMA MODEL AND PHASE TRANSITION}

The linear sigma model Lagrangian can be written as,

$$
\mathcal{L}=\frac{1}{2}\left(\partial_{\mu} \Phi\right)^{2}-\frac{\lambda}{4}\left(\Phi^{2}-v^{2}\right)^{2}+H_{\sigma}
$$

where chiral degrees of freedom are the $\mathrm{O}(4)$ fields $\phi_{a}=(\sigma, \vec{\pi})$. In eq.1 $H_{\sigma}$ is the explicit symmetry breaking term. This term is responsible for finite pion mass. The parameters of the model $\lambda, v$ and $H$ can be fixed using the pion decay constant $f_{\pi}$, and $\sigma$ and pion masses. With standard parameters, $f_{\pi}=92 \mathrm{MeV}, m_{\sigma}=600 \mathrm{MeV}$ and $m_{\pi}=140$ $\mathrm{MeV}$, one obtain,

$$
\lambda=\frac{m_{\sigma}^{2}-m_{\pi}^{2}}{2 f_{\pi}^{2}} \sim 20, v^{2}=f_{\pi}^{2}-\frac{m_{\pi}^{2}}{\lambda}=(87 M e V)^{2}, H=f_{\pi} m_{\pi}^{2}=(122 M e V)^{3}
$$

In eq.1, the potential corresponds to zero temperature. At finite temperature (T), to leading order in $\lambda$, thermal fluctuations $<\delta \phi^{2}>$ of the pions and sigma mesons generate an effective Hartree type dynamical mass giving rise to an effective temperature dependent potential. In the high temperature expansion, this results in 19],

$$
m_{t h}^{2} \rightarrow \frac{\lambda}{2} T^{2}
$$

As told in the beginning, if in heavy ion collision, a certain region undergoes chiral symmetry restoration, that region must be in contact with some environment or background. Exact nature of the environment is difficult to determine but presumably it will be consists of mesons and hadron (pions, nucleon etc.). Recognising the uncertainty in the exact nature of the environment, we choose to represent it by a white noise source, i.e. a heat bath. To analyse 
the effect of environment or heat bath on the possible disoriented chiral condensate, we propose to study Langevin equation for linear $\sigma$ model. Langevin equation for $\mathrm{O}(4)$ fields has been used previously to investigate the interplay of noise and dissipation on the evolution and stability DCC. Recently it has been shown that in the $\phi^{4}$ theory, hard modes can be integrated out on a two loop basis resulting in a Langevin type equations for the soft modes [21,22], with dissipation and noise. The resulting equation is non-local in time and quite complicated. If a Markovian limit exists to this equation, then one may hope to obtain a simpler equation (as the present equation) which can be used for practical purposes.

We write the Langevin equation for linear $\sigma$ model as,

$$
\left[\frac{\partial^{2}}{\partial \tau^{2}}+\left(\frac{1}{\tau}+\eta\right) \frac{\partial}{\partial \tau}-\frac{\partial^{2}}{\partial x^{2}}-\frac{\partial^{2}}{\partial y^{2}}-\frac{1}{\tau^{2}} \frac{\partial^{2}}{\partial Y^{2}}+\lambda\left(\Phi^{2}-f_{\pi}^{2}-T^{2} / 2\right)\right] \Phi=\zeta(\tau, x, y, Y)
$$

where $\tau$ is the proper time and $\mathrm{Y}$ is the rapidity, the appropriate coordinates for heavy ion scattering. To be consistent with fluctuation-dissipation theorem, we have included a dissipative term $\eta$ in the equation. As told earlier, the environment or the heat bath $(\zeta)$ was represented as a white noise source with zero average and correlation as demanded by fluctuation-dissipation theorem,

$$
\begin{aligned}
<\zeta(\tau, x, y, Y) & >0 \\
<\zeta_{a}(\tau, x, y, Y) \zeta_{b}\left(\tau^{\prime}, x^{\prime}, y^{\prime}, Y^{\prime}\right)> & =2 T \eta \frac{1}{\tau} \delta\left(\tau-\tau^{\prime}\right) \delta\left(x-x^{\prime}\right) \delta\left(y-y^{\prime}\right) \delta\left(Y-Y^{\prime}\right) \delta_{a b}
\end{aligned}
$$

where a,b corresponds to $\pi$ or $\sigma$ fields. The noise term will continuously heat the system, while the dissipative term will counteract it. Equilibrium is achieved when the system is thermalised at the temperature dictated by the heat bath. We note that eq.11 can not be derived formally, but as will be shown below, it does describe the equilibrium physics correctly. Thus at least on phenomenological level, its use can be justified, and we can hope that it will describe correctly the non-equilibrium physics, as required for the DCC phenomena.

Before we proceed further few words are necessary about the applicability of Langevin equation in describing DCC, when the noise term contains temperature. DCC is basically a non-equilibrium phenomena. By using temperature, we are approximating it as a equilibrium one. This approximation is valid when the system is not far from equilibrium. Fluctuation-dissipation relation is also valid for such a system only. In such a system, it is possible to define a temperature at each point of time, if the time scale of the constituents of the system is large compared to the time scale of the collective variable (in this case expansion of the system).

Set of partial differential eqs. 1 1 were solved on a $32^{3}$ lattice using a lattice spacing of 1 fm and using periodic boundary conditions. Solving eqn.1 require initial conditions $(\phi$ and $\dot{\phi})$. We distribute the initial fields according to a random Gaussian with,

$$
\begin{aligned}
<\sigma> & =(1-f(r)) f_{\pi} \\
<\pi_{i}> & =0 \\
<\sigma^{2}>-<\sigma>^{2}=<\pi_{i}^{2}>-<\pi_{i}>^{2} & =f_{\pi}^{2} / 4 f(r) \\
<\dot{\sigma}> & =<\dot{\pi}_{i}>=0 \\
<\dot{\sigma}^{2}>=<\dot{\pi}>^{2} & =f_{\pi}^{2} f(r)
\end{aligned}
$$

The interpolation function

$$
\left.f(r)=\left[1+\exp \left(r-r_{0}\right) / \Gamma\right)\right]^{-1}
$$

separates the central region from the rest of the system. We have taken $r_{0}=11 \mathrm{fm}$ and $\Gamma=0.5 \mathrm{fm}$. The initial field configurations corresponds to quench like condition [1,2] but it is important to note that the field configuration at equilibrium will be independent of initial configuration. The other parameter of the model is the friction. The friction coefficient was assumed to be $\eta=\eta_{\pi}+\eta_{\sigma}$, where $\eta_{\pi, \sigma}$ are the friction coefficient of the pion and the sigma fields. They have been calculated by Rischke [28],

$$
\begin{aligned}
& \eta_{\pi}=\left(\frac{4 \lambda f_{\pi}}{N}\right)^{2} \frac{m_{\sigma}^{2}}{4 \pi m_{\pi}^{3}} \sqrt{1-\frac{4 m_{\pi}^{2}}{m_{\sigma}^{2}}} \frac{1-\exp \left(-m_{\pi} / T\right)}{1-\exp \left(-m_{\sigma}^{2} / 2 m_{\pi} T\right)} \frac{1}{\left.\exp \left(m_{\sigma}^{2}-2 m_{\pi}^{2}\right) / 2 m_{\pi} T\right)-1} \\
& \eta_{\sigma}=\left(\frac{4 \lambda f_{\pi}}{N}\right)^{2} \frac{N-1}{8 \pi m_{s} i g m a} \sqrt{1-\frac{4 m_{\pi}^{2}}{m_{\sigma}^{2}}} \operatorname{coth} \frac{m_{\sigma}}{4 T}
\end{aligned}
$$


Equilibrium physics will be independent of the exact value of the friction also. Friction just determine the rate of approach towards the equilibrium.

To show that equilibrium physics is independent of the initial field configurations, in fig.1, we have shown the (proper) time evolution of the condensate value of $\sigma$ field,

$$
<\sigma>=\frac{1}{V} \int \sigma d x d y d Y
$$

for two different initial conditions. In one of them, the initial configuration was chosen such that $<\operatorname{sigma}>\sim f_{\pi}$, (depicted in fig.1 as white symbol) and in the other the initial configuration was chosen such that $<$ sigma $>\sim 0$ (depicted in the figure by the filled symbol). We have shown the evolution of the condensate for two fixed temperature of the heat bath, $20 \mathrm{MeV}$ and $40 \mathrm{MeV}$. Equilibrium configuration is reached about $10 \mathrm{fm}$ of evolution. For both the temperatures, the equilibrium condensate value is independent of the initial value. It is also noted that the at higher temperature, the $\langle\sigma\rangle$ condensate (as expected) settles to a lower value.

Equilibrium configuration is also independent of the exact value of the friction. In fig.2, we have plotted the evolution of $\sigma$ condensate for two different values of the friction; $\eta$ as given in eq.5 and half its value. Here again we perform the calculation for two different temperatures of the heat bath, 20 and $40 \mathrm{MeV}$. It can be seen the equilibrium value of the condensate donot depend on the exact value of the friction. Friction determines the rate at which equilibrium is achieved.

Having thus shown that the equilibrium value of the fields are independent of the initial conditions or the exact value of the friction, we now proceed to study the phase transition in the model. To this end, we keep the heat bath at fixed temperature $(\mathrm{T})$ and evolve the fields for sufficiently long time such that equilibrium condition is reached. The condensate value of the $\sigma$ field at equilibrium can be considered as the order parameter for the chiral phase transition. In the symmetry broken phase it will have non-zero value while in the symmetric phase it will vanish. We note that for symmetry restoration phase transition the order parameter should exactly vanish for temperatures $\geq T_{c}$ [29]. Small but non-zero value of the condensate will indicate approximate symmetry restoration.

In fig.3, we have shown the equilibrium condensate value of the sigma field at different temperatures. With the explicit symmetry breaking term in 1 the pions are massive. Thus one should not get exact symmetry restoration phase transition in the model. Indeed, in our simulation also, exact phase transition is not obtained. At low temperature condensate value as expected is around $f_{\pi}$, and it decreases with temperature. However, though it became very small, it donot vanish even at very large temperature. Thus there is no exact phase transition in the model. However very small value of $\langle\sigma\rangle$ at high temperature in comparison to its zero temperature value, indicate that there is partial restoration of the symmetry.

If the explicit symmetry breaking term is neglected, then there is exact chiral symmetry in the model. In fig.4, we have presented the equilibrium condensate values of the sigma field as a function of temperature for zero mass pions. Now we find that sigma condensate exactly vanishes for temperature higher $120 \mathrm{MeV}$. The model shows that with zero mass pions, there is a symmetry restoring phase transition at high temperature.

\section{DISORIENTED CHIRAL CONDENSATE DOMAIN FORMATION}

In the last section, we have shown that the Langevin equation 1 correctly reproduces the equilibrium behavior of the linear $\sigma$ model fields. For zero mass pions, simulation of the Langevin equations shows symmetry restoring phase transition at appropriate temperature. For massive pions it was shown that, though there is no exact symmetry restoring phase transition, nevertheless symmetry is restored to a great extent.

Total or partial chirally symmetric phase (as is the case for zero or finite pion mass) at high temperature will roll back to symmetry broken phase as the system cools and temperature drops below the transition temperature. As told earlier, it is has been conjectured [1.2] that during the roll down period, pseudo scalar condensate $\left\langle\bar{q} \tau \gamma_{5} q>\right.$ can assume non vanishing values, (instead of of remaining zero as in the ground state). In the process domain like structure with definite isospin orientation may emerge during the roll down period. Numerical simulations of linear sigma model with quench like initial field configurations shows domain like structures [14]. It was also seen that $\pi \pi$ correlation is also increased [14. However, in heavy ion collision, quench is not a natural initial condition. $\langle\phi\rangle$ and $<\dot{\phi}>$ are in a configuration appropriate for high temperature but that of $\left\langle\phi^{2}>\right.$ and $<\dot{\phi}^{2}>$ are characteristic of a lower temperature. On the contrary, thermalised fields are better suited to mimic initial conditions that may arise in heavy ion collisions. Here, $\langle\phi\rangle,\langle\dot{\phi}\rangle$ as well as $\left\langle\phi^{2}\right\rangle$ and $\left\langle\dot{\phi}^{2}\right\rangle$ are in a configuration appropriate for high temperature. 
To see whether domain like structure emerges or not with more appropriate initial condition like the thermalised fields, we proceed as follows: using the Langevin equation 1, we thermalised the fields at temperature $\mathrm{T}=200 \mathrm{MeV}$. Next we use the thermalised fields as the initial configuration and now we allow the heat bath to cool and follow the evolution of the thermalised fields. Evolution of the thermalised fields now will depend on the exact nature of the friction, however we choose to use the same friction as before. We assume the following cooling law for the heat bath,

$$
T(t)=T_{0} \frac{1}{t^{n}}
$$

with $\mathrm{n}=1$, appropriate for $3 \mathrm{~d}$ scaling expansion, and we call it fast cooling law. We have also used $\mathrm{n}=1 / 3$ (slow cooling), appropriate for 1-dimensional scaling expansion for some demonstrative calculations.

Assuming that the number density is proportional to the square of the fields amplitude $\left(N_{\pi} \propto \phi_{\pi}^{2}\right)$, at each lattice point, we calculate the neutral to total pion ratio according to,

$$
f(x, y, Y)=\frac{N_{\pi_{0}}}{N_{\pi_{1}}+N_{\pi_{2}}+N_{\pi_{0}}}
$$

Very large or small value of the ratio, over an extended spatial zone will be definite indication of disoriented chiral condensate domain formation.

\section{A. DCC domains without explicit symmetry breaking term}

We first consider the case for zero mass pions, i.e. neglecting the symmetry breaking term in eq.1. For zero mass pions there is a exact symmetry restoring phase transition, the condensate value of the $\langle\sigma\rangle$ field vanishes around $\mathrm{T}=120 \mathrm{MeV}$. In fig.5, evolution of the neutral to total pion ratio at rapidity $Y=0$, with fast cooling, is shown. The initial distribution (panel a) do not show any domain like structure with very high or low value of the ratio (the distribution was random). No domain like structure is seen even after thermalisation of the fields at $\mathrm{T}=200 \mathrm{MeV}$ (panel b). Large domain like structure with high/low value of the ratio $f$ starts to emerge after 10-15 fm of evolution and cooling. With time domain like structure grow. It seems that indeed disoriented chiral condensate domains are formed in presence of noise and friction, even with thermalised fields. In fig. 6 and 7 , we have shown the same results but at different rapidities. At other rapidities also, similar behaviour is seen. Fig.5-7, clearly demonstrate that with fast cooling law, multiple disoriented chiral condensate domains with definite isospin orientations can form as the chirally symmetric phase roll back to broken phase. However domain formation occur quite late in the evolution. With the scaling cooling law, by the time domain like structures emerges, the system is cooled to $\sim 20 \mathrm{MeV}$. Applicability of classical field theory down to that low temperature is questionable. Apart from that it is doubtful whether in heavy ion collision, system can be allowed to be cooled to this extent. Current wisdom is that the hadrons freeze-out around 100-160 MeV. With this reservation in mind, it may be said that even with thermal fields domains of disoriented chiral condensate can form. But the domain growth occur at late times.

To see whether domain like formation occur if the system is cooled slowly, we have repeated the above calculation with the cooling law corresponding to $1 \mathrm{~d}$ scaling law. In fig.8, contour plot of the neutral to total pion ratio at rapidity $\mathrm{Y}=0$ is shown. It can be seen that in this case there is no extendent zone with very large or small $f$, indicating domain like formation is not likely when the expansion or cooling is slower. Similar results are obtained at other rapidities also. If the cooling is slow then the fields gets enough time to adjust to their new environment, consequently energy can be equipartitioned among all the modes. In heavy ion collision, 1d scaling expansion is more appropriate than 3d scaling expansion. Present simulation then indicate that it is unlikely that large size disoriented chiral condensate domains will be formed in heavy ion collisions.

\section{B. DCC domains with explicit symmetry breaking term}

For finite mass pions, there is no exact phase transition in the model. However, symmetry is partially restored. To see whether DCC domains are formed even when there is no exact phase transition, we repeat the above calculation including the symmetry breaking term. In fig.9, xy contour plot of the neutral to total pion ratio at rapidity $Y=0$ is shown. The cooling was fast. We donot find any domain like configuration. With slower cooling law also, though not shown, we donot find any indication of DCC domains formation. Indeed, if domains are not formed with fast cooling law, then it is unlikely that they will be formed with a slow cooling law. The simulation results indicate that there is no disoriented chiral condensate domains with finite mass pions. 


\section{PION-PION CORRELATION}

We define a correlation function at rapidity $Y$ as [14],

$$
C(r, \tau)=\frac{\sum_{i, j} \pi(i) \dot{\pi}(j)}{\sum_{i, j}|\pi(i)||\pi(j)|}
$$

where the sum is taken over those grid points $i$ and $j$ such that the distance between $i$ and $j$ is $r$. As DCC domains are not formed, irrespective of pion mass with slow cooling law, in the following we will present the correlation results for the fast cooling law only. In fig. 10, we have shown the temporal evolution of the correlation function at rapidity $Y=0$, for finite mass pions. Initially, the thermalised pions have no correlation length beyond the lattice spacing of $1 \mathrm{fm}$. With time correlation length increases. But really large long range correlation builds up quite late in the evolution, after 10-15 fm of evolution. Pions separated by large distances are then correlated. The results are in accordance with the contour plot of the neutral to total pion ratio, showing DCC domain formation for zero mass pions at late times.

With massive pions, results are quite different (fig.11). In this case as shown earlier, there is no exact phase transition. Also, evolution of thermalised fields do not results into large domain like formation. Similarly, we find that there is not much long range correlation even at late stage of the evolution.

\section{PROBABILTY DISTRIBUTION OF NEUTRAL TO PION RATIO}

If a single DCC domain is formed in heavy ion collision, it can be easily detected. Probability to obtain a particular fraction

$$
f=\frac{N_{\pi^{0}}}{N_{\pi^{+}}+N_{\pi^{-}}+N_{\pi^{0}}}
$$

of neutral pion from a single domain is $P(f)=1 / 2 \sqrt{f}$ [1.22]. However, our simulations indicate that if at all domains are formed, there will be quite a few number of domains. Naturally the resultant distribution will not be $1 / \sqrt{f}$ type. In this section we obtain the probability distribution of neutral to total pion ratio.

We calculate the number of pions at rapidity $\mathrm{Y}$ by integrating square of the amplitude of the pion fields over the space-time as,

$$
N_{\pi}(Y)=\int \pi^{2} \tau d \tau d x d y
$$

In fig.12, probability distribution for neutral to total pion ratio, for zero mass pions are shown. In three panels, we have shown the distribution obtained after 10,20 and $30 \mathrm{fm}$ of evolution. If the fields are evolved upto $10 \mathrm{fm}$, then the $f$ distribution is sharply peaked around the isospin symmetric average value of $1 / 3$. At the early stage of the evolution, as indicated in figs.5-7, domain like structures or long range correlations are not developed in individual events. As a consequence of that, the neutral to pion ratio is peaked around the isospin symmetric value of $1 / 3$. If the fields are evolved for longer duration (20 or $30 \mathrm{fm}$ ), average of the distribution remains $1 / 3$, but it gets broadened. As shown earlier, at late stage of the evolution there is definite domain like structure in individual events. Long range correlations also develops. Its effect is seen as the increased width. However, increase in width is not as dramatic as expected from the contour plot of the ratio or the correlation studies. In obtaining the f-distribution, we have integrated the pion amplitudes over the time. And as shown earlier, domain like structure develop only at late times. Thus in a single event also, non-DCC pions will be mixed up with DCC pions.

However, quite different results are obtained if the symmetry breaking term is included in the Langevin equation. The pions are massive then. Probability distribution of neutral to total pion ratio, for massive pions are shown in fig.12. Whether the fields are evolved upto 10,20 or $30 \mathrm{fm}$, the distribution is sharply peaked around the isospin symmetric value of $1 / 3$. We have seen that for massive pions, no large domain like structure or long range correlation develop even at late times. The probability distribution reflect those results.

\section{SUMMARY AND CONCLUSIONS}

In summary, we have studied the disoriented chiral condensate formation in presence of background. We have simulated Langevin equation for linear sigma model with a heat bath (the heat bath represents the background) in 
$3+1$ dimension. It was shown that the model reproduces equilibrium physics correctly. For zero mass pions, the model undergoes 2nd order phase transition at $T_{c} \sim 120 \mathrm{MeV}$. Also the model (correctly) donot show exact phase transition for finite mass pions, even though the symmetry is restored to a great extent at high temperature. It was also shown that, for zero mass pions, if the thermalised fields are cooled down sufficiently rapidly, multiple disoriented chiral condensate domains are formed, while for slow cooling no domain like structure is formed. Long range correlation also develops in case of fast cooling. However, even with sufficient fast cooling, with thermalised fields, domains or long range correlations develop quite late in the evolution (after 10-15 fm of evolution). Probability distribution of neutral to total pion ratio shows that the distribution is not $1 / \sqrt{f}$ type, rather it is a Gaussian with average at the isospin symmetric value of $1 / 3$. The width of the Gaussian increases, when domains are formed.

For massive pions our simulation indicate that thermalised fields donot evolve into domain like structure, irrespective of slow or fast cooling law. No long range correlation is also seen. The probability distribution of neutral to total pion ratio also donot show any broadening of width as was seen for zero mass pions.

To conclude, simulation studies of Langevin equations for linear sigma model indicate that for realistic pions, thermalised fields donot evolve into DCC domain like structure.

\section{ACKNOWLEDGMENTS}

The author gratefully acknowledge the kind hospitality of Centre for Theoretical Physics, MIT and McGill University where part of the work was done.

* e-mail address:akc@veccal.ernet.in

[1] K. Rajagopal and F. Wilczek, Nucl.Phys.B404,577,(1993).

[2] K. Rajagopal and F. Wilczek, Nucl.Phys.B399,395,(1993).

[3] K. Rajagopal, Nucl.Phys.A566,567c(1994).

[4] J. D. Bjorken, Int. J. Mod. Phys. A7,4189(1992).

J. D. Bjorken, Acta Phys. Polon, B23,561(1992)

[5] K. L. Kowalski and C. C. Tayolor, Disoriented Chiral Condensate: A white Paper for the Full Acceptance Detector, CWRUTH-92-6 (1992), hep-ph/9211282.

[6] J. D. Bjorken, K. L. Kowalski and C. C. Taylor, SLAC-PUB-6109 (1993), K.L. Kowalski K. L. and Taylor C. C., SLACPUB-6109 (1993), hep-ph/9309235.

[7] J. D. Bjorken, Acta Phys. Polon. B28,2773 (1997).

[8] A. A. Anselm and M. G. Ryskin , Phys. Lett. B 266,482 (1991), A. A. Aneslm, Phys. Lett. B217, 169(1988).

[9] C. M. G. Lattes, Y. Fujimoto and S. Hasegawa, Phys. Reports, 154,247(1980).

[10] J. P. Blaizot ,A. Krzywicki , Phys. Rev.D46, 246 (1992).

[11] I. V. Andreev, JETP Lett. 33, 367 (1981).

[12] J. Randrup, Phys. Rev D55,1188 (1997). J. Randrup, Nucl. Phys. A616,531 (1997). J. Randrup and R. L. Thews, Phys. Rev. D56,4392(1997). J. Schaffner-Bielich and Jorgen Randrup, Phys. Rev. C59,3329 (1999). M. Bleicher, J. Randrup, R. Snellings, X. N. Wang, Phys. Rev. C62 (2000)041901.

[13] Gavin S., Gocksch A. and Pisarski R. D., Phys. Rev. Lett.72 2143, (1994). Gavin S. and Muller B. Phys. Lett.B329 486 (1994).

[14] M. Asakawa, Z. Huang and X.N. Wang, Nucl. Phys. A590, 575c (1995). M. Asakawa, Z. Huang and X.N. Wang, hepph/9408299, Phys. Rev. Lett. 74 (1995) 3126. M. Asakawa, H. Minakata and B. Muller, Phys.Rev.D58, 094011(1998). M. Asakawa, H. Minakata and B. Muller, Nucl. Phys. A638,443c (1998).

[15] J. I. Kapusta and A. P. Vischer, Z. Phys. C75,507 (1997).

[16] C. Chow and T. D. Cohen, nucl-th/9908013, Phys. Rev. C60,054902 (1999).

[17] L. P. Csernai, P. J. Ellis, S. Jeon and J. I. Kapusta, nucl-th/9908020, Phys. Rev. C61,056901 (2000)

[18] A. Krzywicki, J, Serreau, Phys. Lett. B448,257 (1999).

[19] A. Bochkarev and J. Kapusta, Phys. Rev. D54 4066 (1996).

[20] T. S. Biro and C. Greiner, Phys. Rev. Lett. 79, 3138(1997).

[21] C. Greiner and B. Müller, Phys. rev. D 551026 (1997).

[22] Z. Zu and C. Greiner, hep-ph/9910562, Phys. Rev. D 62036012 (2000).

[23] A. K. Chaudhuri, nucl-th/9809018, Phys. Rev. D. 59117503 (1999).

[24] A. K. Chaudhuri, hep-ph/9904269, J. Phys. (in press). 
[25] A. K. Chaudhuri, hep-ph/9908376.

[26] A. K. Chaudhuri, hep-ph/0007332.

[27] A. K. Chaudhuri, hep-ph/0011003.

[28] D. H. Rischke, Phys. Rev. C, 2331 (1998).

[29] L. D. Landau and E. M. Lifshitz, in Statistical Physics, 3rd ed.Pergamon Press, 1993.

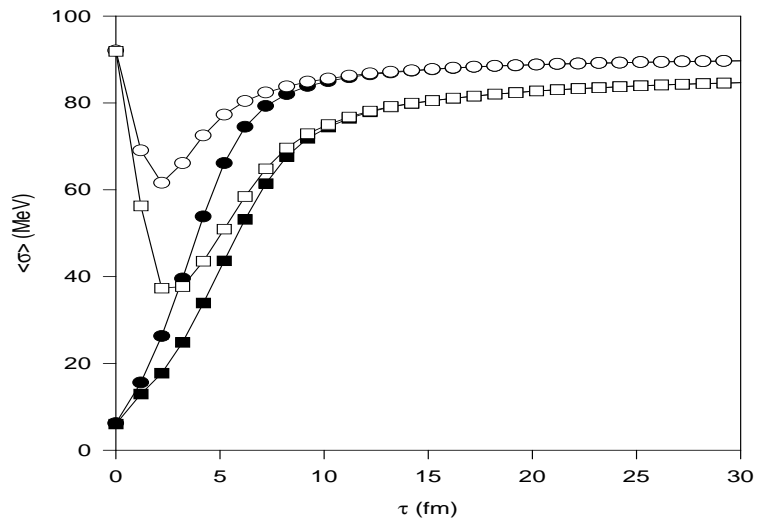

FIG. 1. Evolution of the $\sigma$ condensate with (proper) time for two different fixed temperature $20 \mathrm{MeV}$ and $40 \mathrm{MeV}$ of the heat bath. Two different initial condition evolve to the same condensate value (see text). 


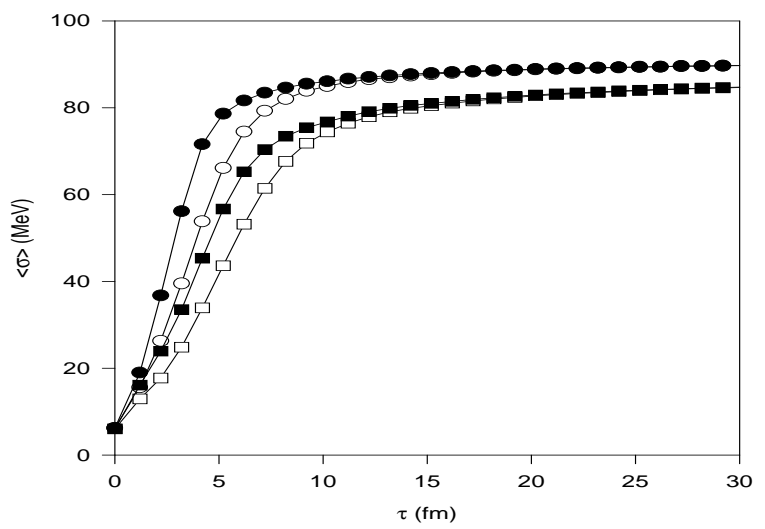

FIG. 2. Evolution of the $\sigma$ condensate with (proper) time for two different fixed temperature $20 \mathrm{MeV}$ and $40 \mathrm{MeV}$ of the heat bath, showing that equilibrium value is independent of friction constant. Two different friction constant results into same equilibrium value (see text).

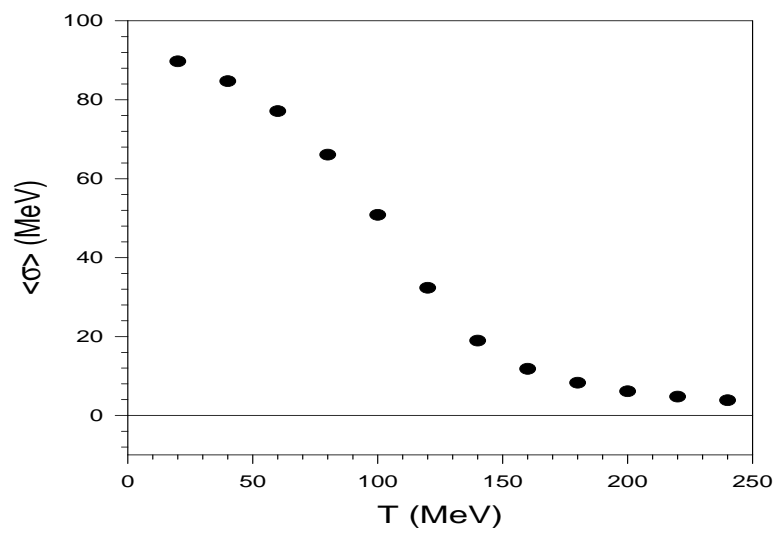

FIG. 3. Equilibrium value of the $\sigma$ condensate as a function of temperature. The pions are massive. 


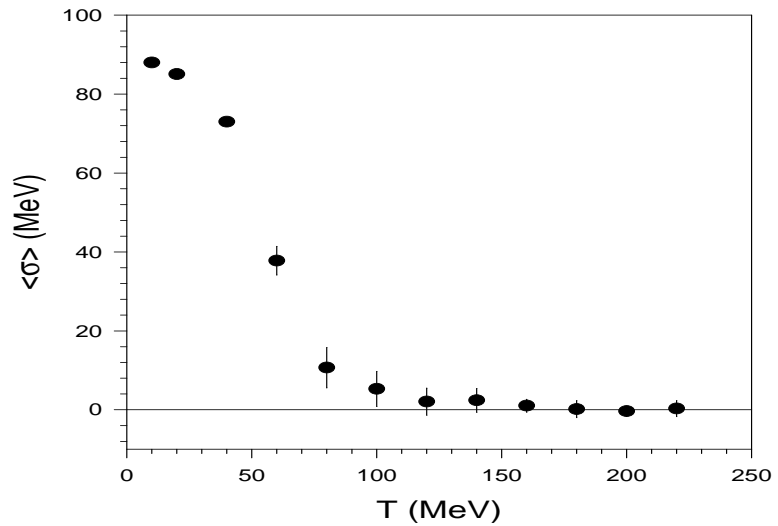

FIG. 4. Equilibrium value of the $\sigma$ condensate as a function of temperature. The pions are massless.

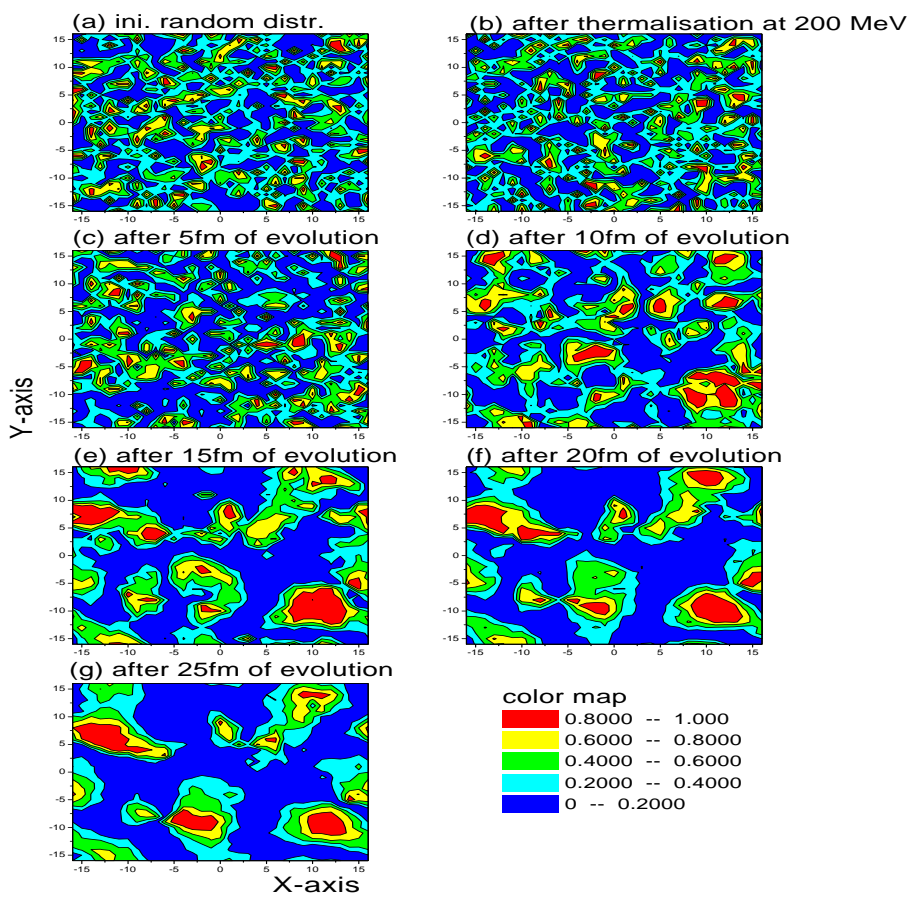

FIG. 5. Contour plot of the neutral to total pion ratio, at rapidity $\mathrm{Y}=0$. The explicit symmetry breaking term is omitted i.e. pions are massless. The cooling law corresponds to fast cooling law. Different panels shows the evolution of the ratio at different times. Domain like structure is evident at late times. 

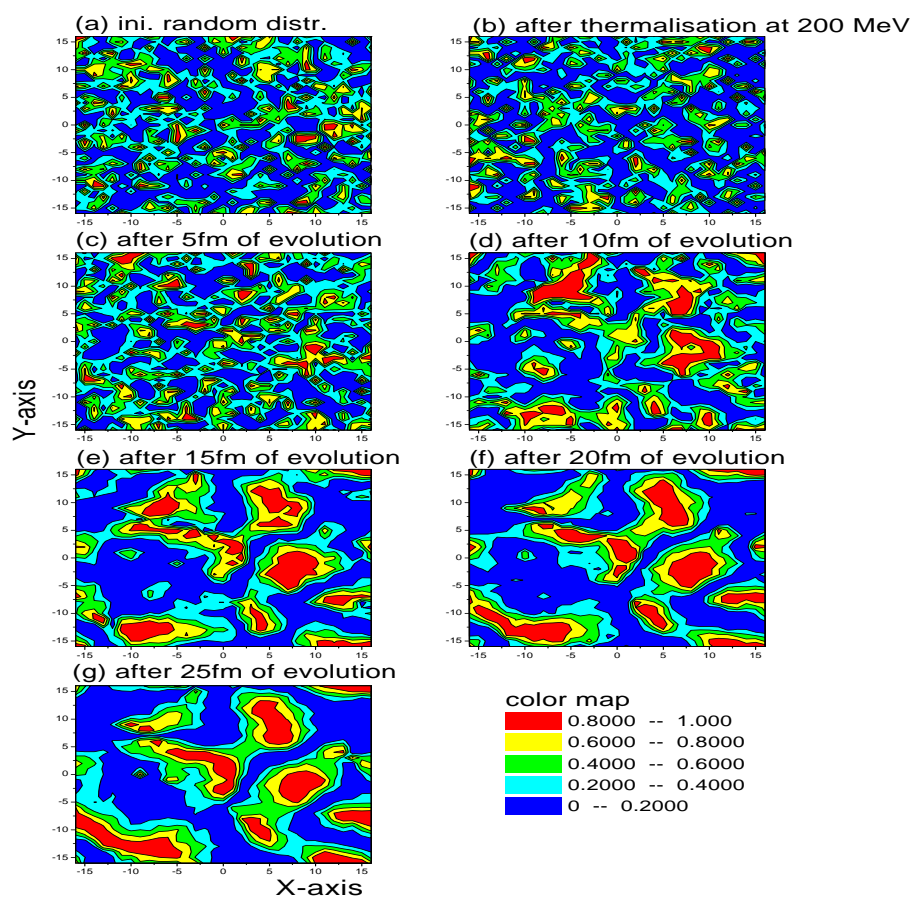

FIG. 6. Same as fig.5, but for rapidity $Y=-4$.
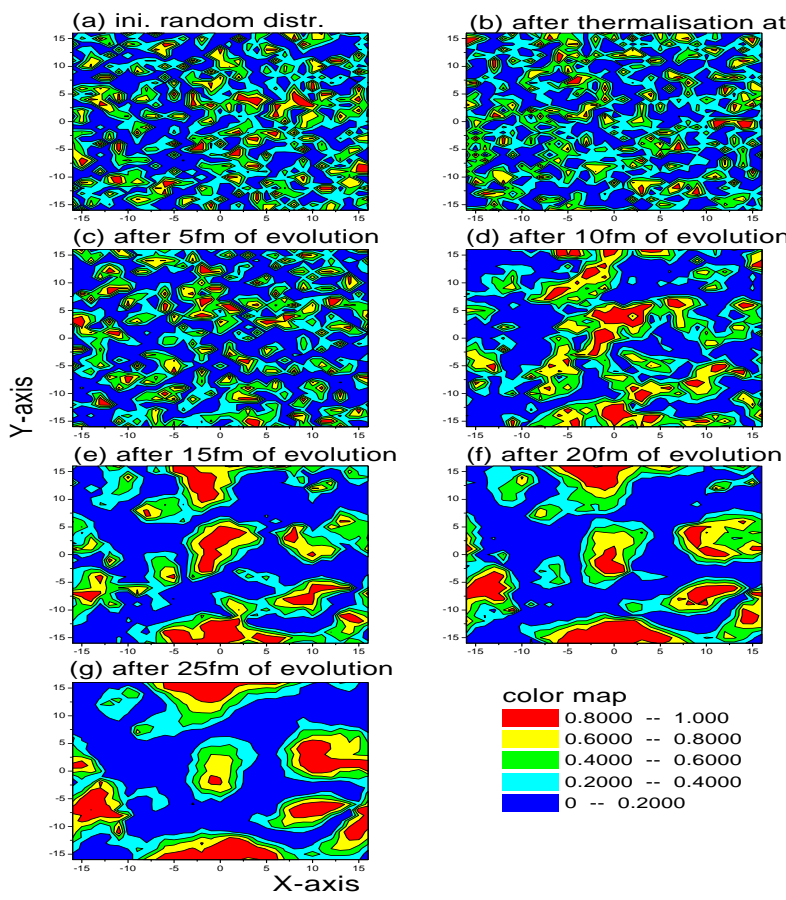

FIG. 7. Same as fig. 5 but for rapidity $\mathrm{Y}=4$. 

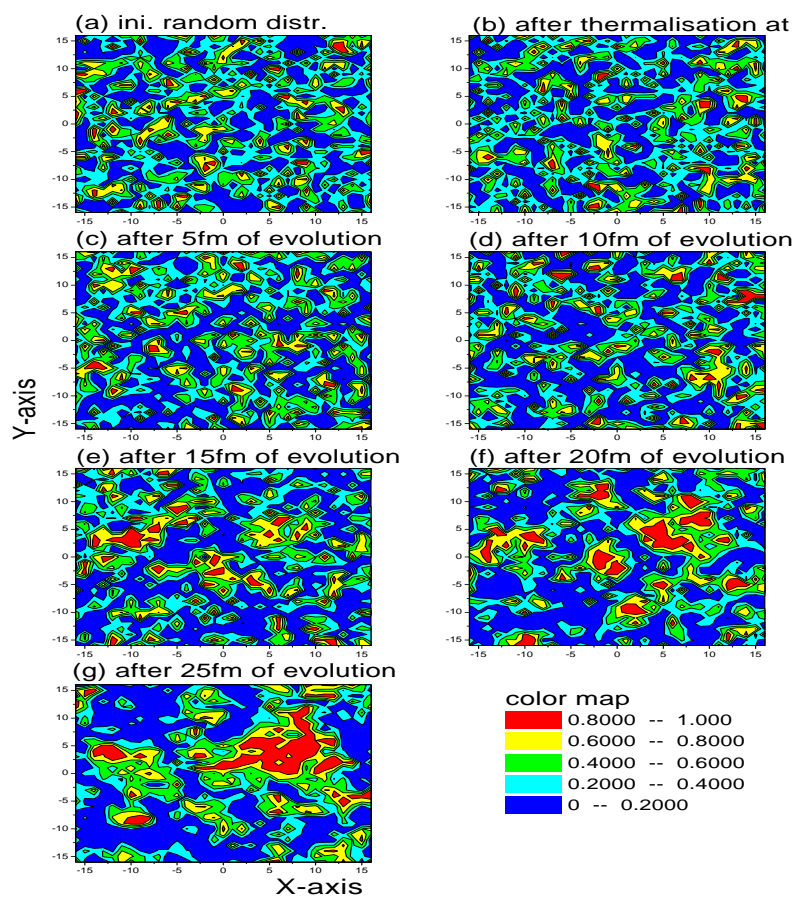

FIG. 8. Contour plot of the neutral to total pion ratio, at rapidity $\mathrm{Y}=0$. The explicit symmetry breaking term is omitted i.e. pions are massless. The cooling law corresponds to slow cooling law. Different panels shows the evolution of the ratio at different times. Domain like structure is evident at very late times.
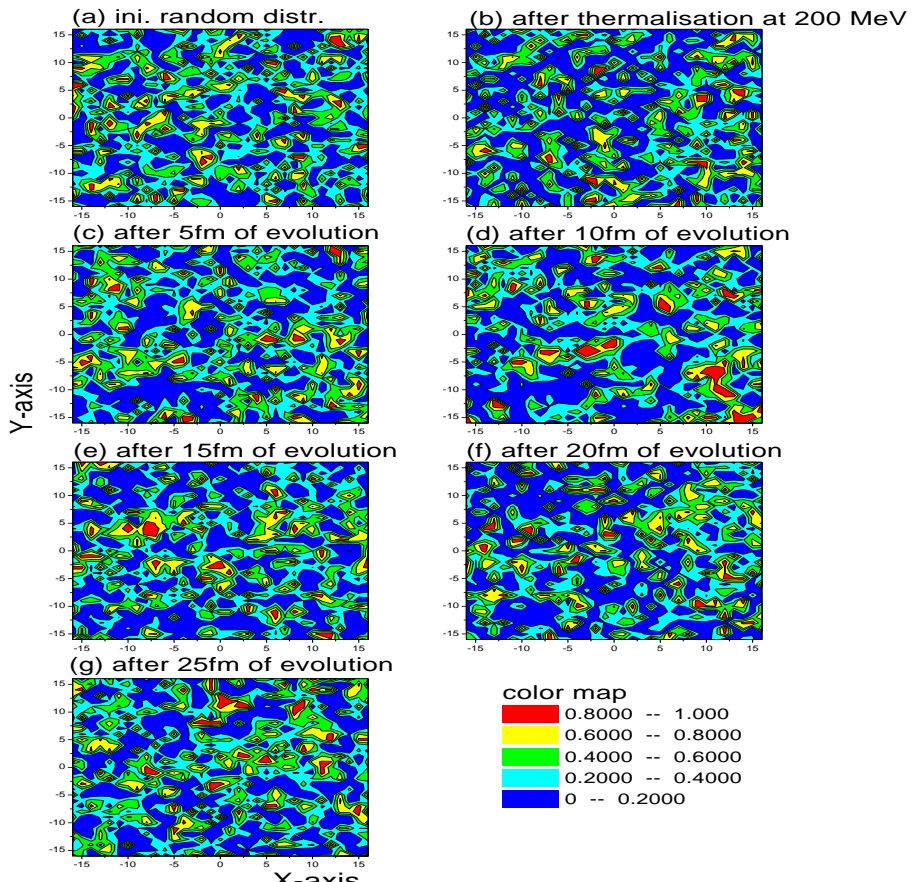

FIG. 9. Contour plot of the neutral to total pion ratio, at rapidity $\mathrm{Y}=0$. The explicit symmetry breaking term is included i.e. pions are massive. The cooling law corresponds to fast cooling law. Different panels shows the evolution of the ratio at different times. No Domain like structure is evident even at late times. 


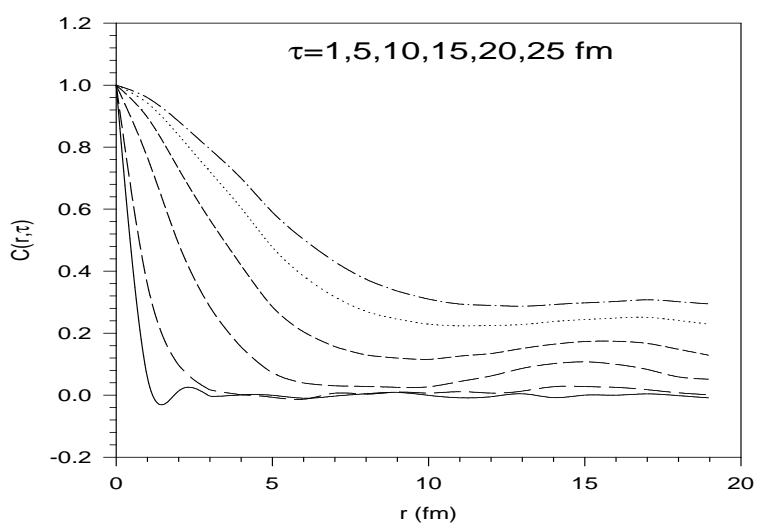

FIG. 10. Evolution of the correlation function with time for zero mass pions at rapidity Y=0. Cooling law corresponds to fast cooling law. Long range correlation develops at late times.

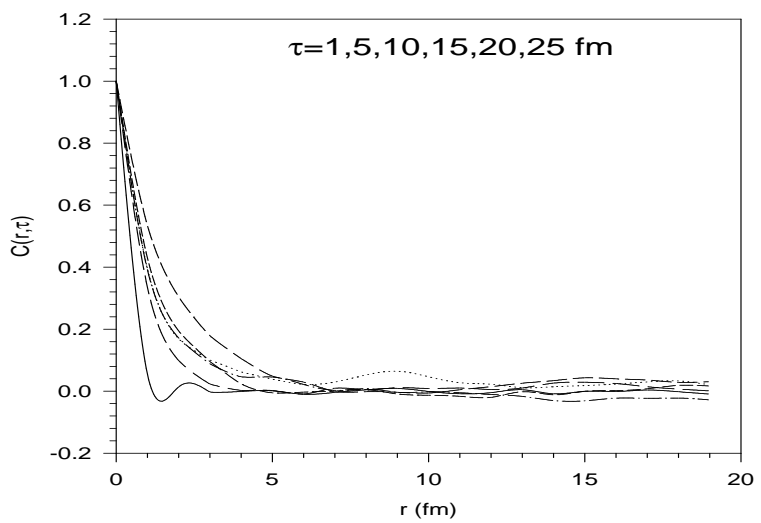

FIG. 11. Evolution of the correlation function with time for massive pions at rapidity $\mathrm{Y}=0$. The cooling law corresponds to fast cooling law. No long range correlation develops at late times. 


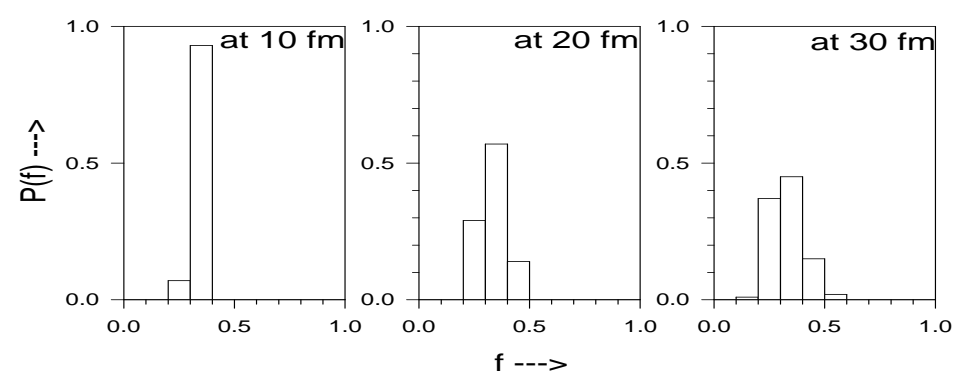

FIG. 12. Probability distribution for the neutral to total pion ratio, at different times. The symmetry breaking term is omitted. The cooling law corresponds to fast cooling law.

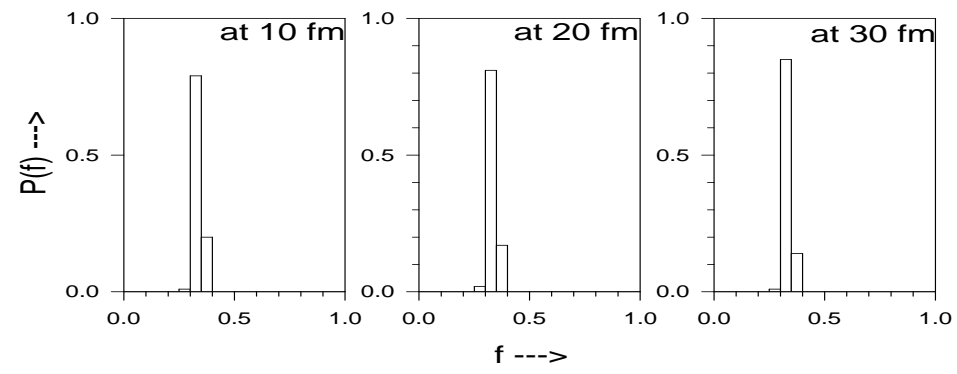

FIG. 13. Probability distribution for the neutral to total pion ratio, at different times. The symmetry breaking term is included. The cooling law corresponds to fast cooling law. 